\title{
Novel surgery for refractory mixed constipation: Jinling procedure - technical notes and early outcome
}

\author{
Weiwei Ding, Jun Jiang, Xiaobo Feng, Anlong Yao, Lin Wang, Jieshou Li, Ning Li
}

Research Institute of General Surgery, Jinling Hospital, Nanjing University School of Medicine, Nanjing, Jiangsu Province, China

Submitted: 13 March 2013

Accepted: 28 May 2013

Arch Med Sci 2014; 10, 6: 1129-1134

DOI: 10.5114 /aoms.2014.47824

Copyright ( 2014 Termedia \& Banach

\section{Abstract}

Introduction: The treatment of slow-transit constipation combined with outlet obstruction is controversial. This study introduced a new surgical strategy, subtotal colectomy combined with a modified Duhamel procedure (Jinling procedure), of which the safety and satisfactory rate were examined. Material and methods: Ninety patients with refractory slow-transit constipation associated with outlet obstruction were consecutively included between Jan 2010 and Dec 2010. All underwent the laparoscopic-assisted Jinling procedure, which added a new side-to-side anastomosis to the colorectal posterior anastomosis after subtotal colectomy. The pre- and post-operative data were collected.

Results: There was no surgery-related death. A total of 39 complications and adverse events were reported in 22 patients (morbidity rate of $24.4 \%$ ). Most complications were managed conservatively without significant events. The satisfactory rate was $93.1 \%$ at 6-month follow-up.

Conclusions: The Jinling procedure is safe for refractory slow-transit constipation associated with outlet obstruction, with minimal major complications and a high satisfaction rate. However, this procedure requires rigorous preoperative examination, exquisite surgical and laparoscopic techniques and excellent perioperative management. The pelvic floor, especially the presacral space, is damaged, and therefore it may be unsalvageable if severe complications, such as anastomosis leakage or ischemia, occur.

Key words: constipation, surgery, safety.

\section{Introduction}

Constipation is a common problem, with up to $20 \%$ of the general population presenting this symptom according to the literature [1-5]. A thorough work-up must be performed in order to categorize the constipation in one of three major groups: (a) slow transmit constipation (STC), (b) outlet obstruction syndrome (ODS), or (c) mixed refractory functional constipation.

An increasing number of patients with constipation suffer from STC associated with concomitant ODS [6]. Of 541 cases of chronic constipation, reported by Ragg [6], nearly one third were classified as outlet obstruction plus STC. Most patients with STC have associated outlet obstruction [6]. The optimal treatment of patients with combined STC and ODS is still unclear. Solely stapled transanal rectal resection (STARR) or colectomy with ileorectal anastomosis could not resolve the coexisting
Corresponding author:

Ning Li MD

Research Institute

of General Surgery

Jinling Hospital

Nanjing University School

of Medicine

305 East Zhongshan Road

Nanjing 210002

Jiangsu Province, China

Phone: 862580860037

E-mail: liningrigs@163.com 


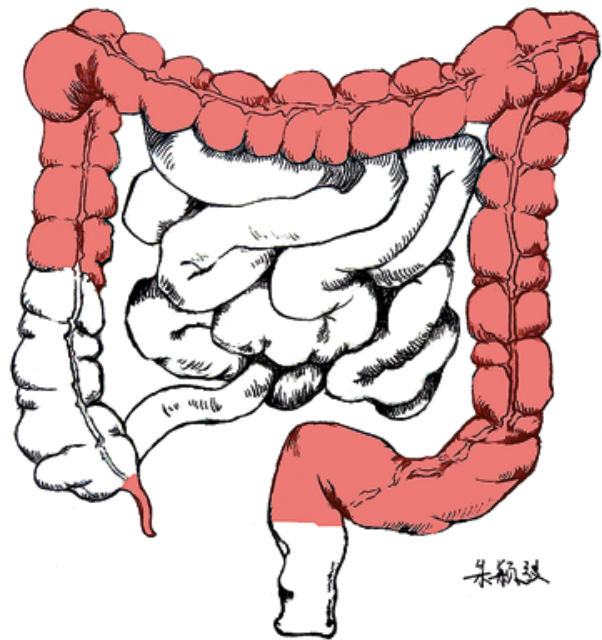

Figure 1 . The resection range of the Jinling procedure: subtotal colectomy and appendectomy

problems $[1,7]$. Inspired by the modified Duhamel operation, in this study we introduced a new surgical procedure, named the Jinling procedure after our hospital, to treat mixed constipation (Figure 1). The whole surgical procedure is described in detail in this study.

\section{Material and methods}

\section{Patient selection}

Patients who were included in this study had to meet the following criteria: (1) meeting the Rome III Constipation Criteria for chronic constipation [8]; (2) having a long disease history (over 6 years) and the quality of life is severely jeopardized and patients expressed a strong need to undergo sur-

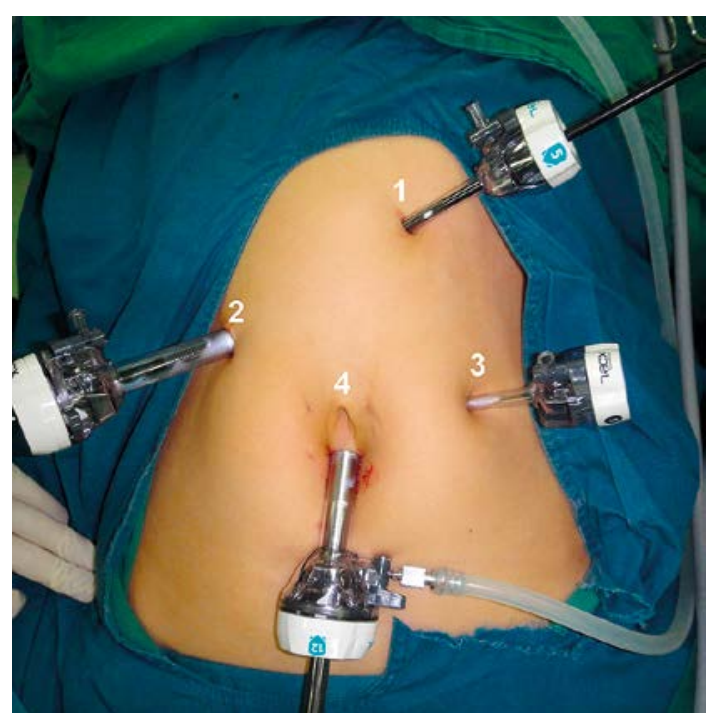

Figure 2. The position of trocars: (1) a 5-mm port is placed between the xiphoid and umbilicus; (2) one additional $12-\mathrm{mm}$ working port is placed on the right side; (3) a 5-mm port is used in the lateral left flank; (4) a 12-mm port for the camera is placed caudally to the umbilicus gical interventions; (3) conservative treatment with diet (1.5 l/day water and high-fiber diet), laxatives (including saline, osmotic and stimulant laxatives, etc.), enemas and biofeedback had been tried over the past 2 years without success; (4) a thorough examination is undertaken to conform the coexistence of ODS and STC. The diagnosis of ODS is based on the evidence of anatomical defects (internal rectal prolapse and/or rectocele, assessed clinically and confirmed by dynamic imaging), and functional abnormalities (puborectalis dysfunction and/or pelvic floor dyssynergy as assessed by electromyography and anorectal manometry). The STC was defined as retention of at least $20 \%$ of a single ingestion of 20 radio-opaque markers (SGmark) in the colon over $120 \mathrm{~h}$. Colonic distribution of markers indicated the coexistence of colonic inertia and outlet obstruction.

Exclusion criteria included patients with a contraindication to general anesthesia, immunocompromised status, physical or psychological problems precluding data collection, inflammatory bowel disease, or septic conditions of the anorectum.

\section{Surgical details}

From January 2010 to Decemeber 2010, 90 patients received the Jinling procedure; about $96 \%$ came from other cities in China. As a national cutting-edge gastroenterology surgical center, Jinling Hospital, a tertiary teaching hospital with 2000 beds, receives patients from nationwide and worldwide. All patients were operated on by the same surgical team. Mechanical bowel preparation consisted of two $(45 \mathrm{ml})$ bottles of oral sodium phosphate and two $(133 \mathrm{ml})$ bottles of sodium phosphate rectal solution (Fleet Enema) the day before surgery. In all patients we administered antibiotic prophylaxis with $1 \mathrm{~g}$ of ceftriaxone and $500 \mathrm{mg}$ of metronidazole i.v. $0.5 \mathrm{~h}$ before the procedure.

The patient was catheterized and placed in a supine Trendelenburg position of about $30^{\circ}$. This position was held with a mild lateral rotation (to the right during left colon dissection and to the left during right colon dissection). A pneumoperitoneum with intra-abdominal pressure of $12 \mathrm{~mm} \mathrm{Hg}$ was established according to an "open technique" with a Hasson trocar. A $30^{\circ}$ camera was used. A $12-\mathrm{mm}$ port is placed just cephalad of the umbilicus. A 5-mm port is used in the lateral left flank. One additional 12-mm working port is placed on the right side, and another $5-\mathrm{mm}$ port is placed between the xiphoid and umbilicus (Figure 2). Ultrasonic shears are then used to take down the short gastric and epiploic vessels.

A lateral to medial approach was used to mobilize the entire colon. The mesocolon was divided using bipolar cautery (Ligasure, ValleyLab, Boulder, CO) with careful identification and division of the 
left, middle and right colonic vessels. The operation started with mobilization of the hepatic flexure. After dissection of the great omentum from the transverse colon and mesocolon followed by complete mobilization of the descending colon with identification of the left ureter and gonadic vessels, a transverse surgical incision (about 5-7 cm) was performed (Figure 3). We prefer to make a short transverse abdominal incision instead of the whole laparoscopic procedure, because abdominal lavage with a large amount of solution (200 ml/kg body weight) can be performed, and the mesenteric hiatus after removing the colon can be closed, and the specimen can be easily pulled through the incision.

After the extraction of the entire colon out of the abdominal cavity, protecting the abdominal wall with a plastic ring drape, appendectomy and colonic resection preserving about $10-12 \mathrm{~cm}$ of ascending colon from the ileocecal valve was performed. The actual length of remaining ascending colon was individually determined by the blood supply of the ileocolic artery. After careful resection of the fatty appendages and preservation of the vascular pedicles close to the bowel, the anvil of a $29 \mathrm{~mm}$ circular stapler was installed at the ascending colon stump by a purse string. It is essential to make sure the colon stump can move downwards to the pelvis. Rapid dissection of the presacral space was finished with electricity knife, with special care to preserve the sacral nerves. The rectum was transected at the level of the promontory with a 60-mm linear stapler, leaving a 9-10 cm-long rectal stump (Figure $4 \mathrm{~A}$ ). The rectal stump was sewn in a two-layer maneuver. The anastomosis site was punctured using surgical forceps at the posterior wall of the rectum (1.5-2 cm above the dentate line). A 14 Fr red rubber tube was squeezed by the forceps peritoneally and pulled out through the anus. After connecting the tube to the stapler and manual dilation of the anus, the stapler was introduced to the anus and rectum stump correctly. An end-to-side colorectal posterior anastomosis was performed (Figure $4 \mathrm{~B}$ ). Finally, a 60-mm linear cutter (ECHELON, Ethicon Endo-Surgery) was inserted into the rectum and distal colon (Figure 4 C) and another side-to-side colorectal anastomosis was performed (creating a 5-6 $\mathrm{cm}$ anastomosis, Figure $4 \mathrm{D}$ ). The abdominal cavity was lavaged with a large amount of warm sterile saline and drained at the pelvis.

\section{Results}

\section{General information}

Between January 2010 and December 2010, 90 patients received laparoscopic-assisted Jinling procedure. There were 67 females and 23 males with a mean age of 48.4 years (range: 18-74

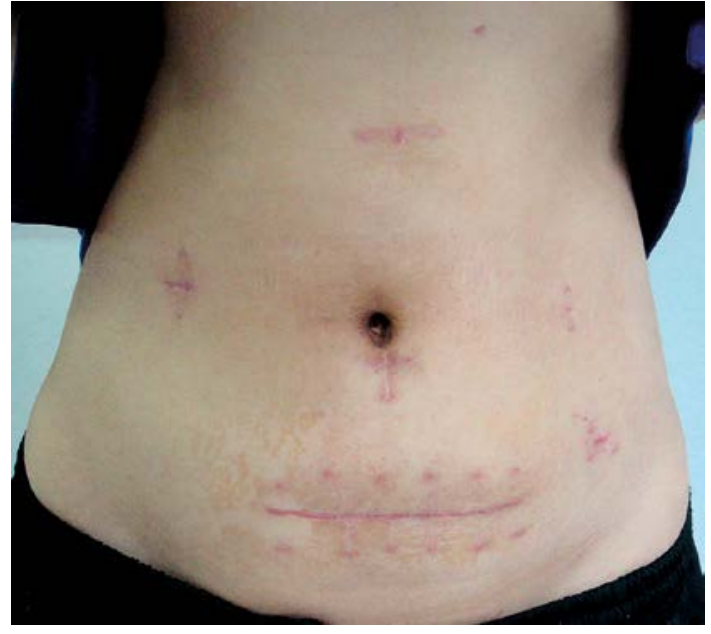

Figure 3. Surgical incision of Jinling procedure

years). Mean disease onset age is 26.9 years old (range: 13-65) with the mean duration of about 132.1 months. Most women had one delivery, and 10 of them were multifarious. Eighteen patients had a previous anal surgical procedure. Mean operating room time was 188 min (range 157-251 $\mathrm{min}$ ). There were 3 conversions to an open procedure $(3.3 \%)$ in patients with intra-abdominal adhesions. The median length of hospital stay was 10.1 days (range: 6-19 days) and of stay after surgery 6.9 days (range: $4-17$ days). The median time to resolution of ileus, defined as time to tolerate oral fluids, was 3 days (range: 1-7 days).

\section{Surgical and safety data}

A total of 39 complications and adverse events were reported in 22 patients, giving an overall morbidity rate of $24.4 \%$. In this group of patients, bowel movement frequency increased from 1.5 \pm 0.6 per week preoperatively to $31.2 \pm 2.1$ per week 1 month and $18.2 \pm 5.9$ per week 3 months postoperatively $(p<0.001)$. Forty-two $(46.7 \%)$ patients developed early postoperative diarrhea within 2 weeks, which was conservatively treated with a dietary fiber supplement, Bifidobacterium, and somatostatin and was not regarded as a major complication. Postoperative urinary retention occurred in 10 (11.1\%) patients. Seven (7.7\%) patients required catheterization within 1 week, and all recovered in 1-2 weeks except 2 patients who recovered after 4 weeks. Seventeen patients (18.9\%) developed gastroparesis and required prolonged placement of a gastric tube until 2 weeks after surgery. Seven (7.8\%) patients reported small intestinal obstruction, 5 patients made a recovery with conservative management and 2 (2.2\%) patients underwent reoperation. Anastomotic bleeding occurred in 5 (5.6\%) patients. Most was minor bleeding (less than $200 \mathrm{ml}$ of blood lost at postoperative day 1), 
A
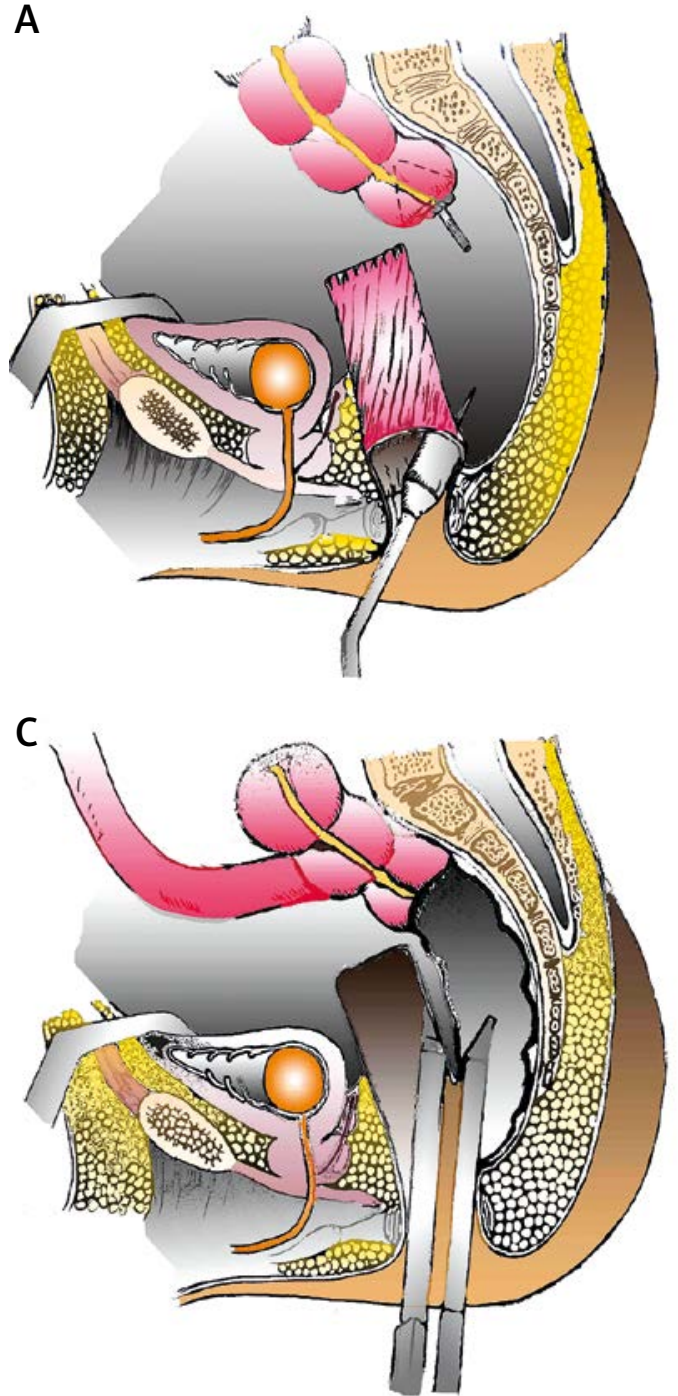

B

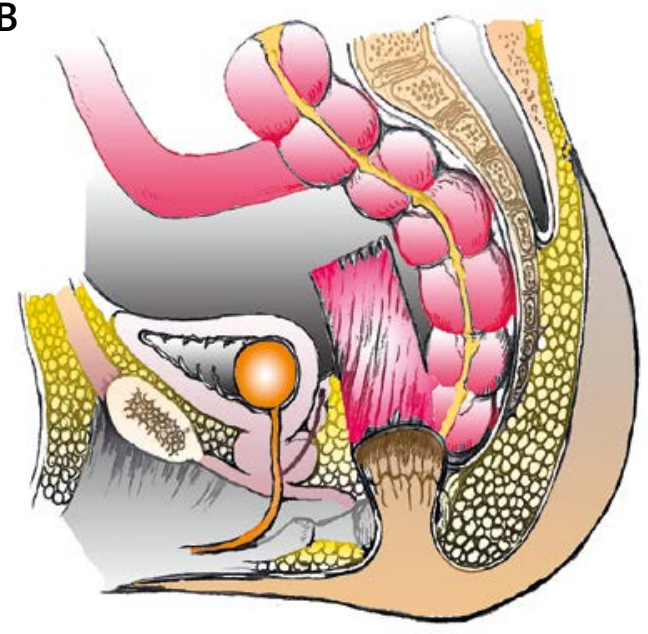

D

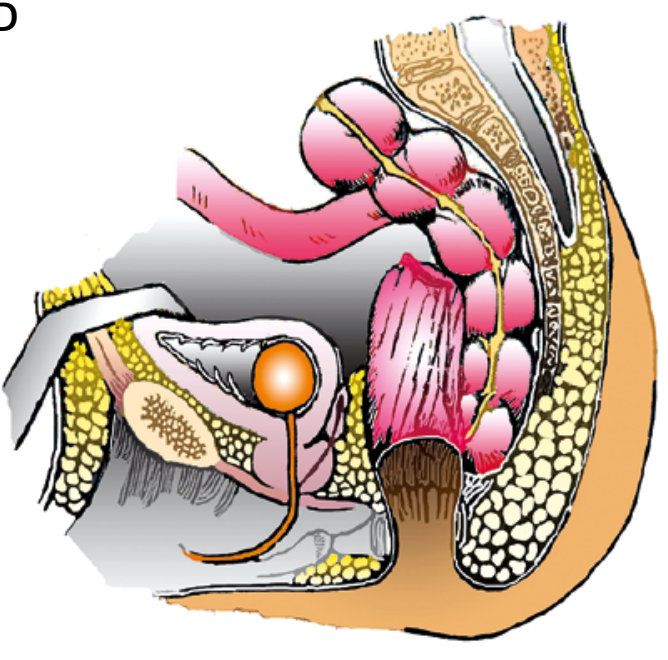

Figure 4. Details of Jinling procedure. A - Circular staplers punctured through the anus at the posterior wall of the rectal stump, about $2 \mathrm{~cm}$ above the dentate line. The anvil of a circular stapler was installed at the ascending colon stump by a purse string. B - An end-to-side colorectal posterior anastomosis was performed. C - Two arms of the linear cutter were inserted into the rectum and ascending colon, respectively. D - The whole Jinling procedure was finished, with a cone-shape anastomosis above the dentate line (drawn by ZHU Wentao, from Nanjing Normal University)

all cured with local compression and pharmacological therapy. No anastomotic leakage occurred. There was no incontinence or surgery-related death in any of the patients.

\section{Discussion}

In this study, we modified the classic subtotal colectomy with colorectal anastomosis (SCCA), and added a new anastomosis to SCCA, aiming to solve the coexistence of ODS and STC. In the Jinling procedure, we chose SCCA, which represents a widely adopted surgical approach for the treatment of STC [9]. In particular, the preservation of the terminal ileum, cecum, and ileocecal valve theoretically represents a physiological advantage over ileorectal anastomosis, avoiding malabsorp- tion and colonic bacterial contamination of the small bowel; moreover, the cecal reservoir and its water-absorbing function would seem to provide an additional preventive factor regarding diarrhea, urgency, and incontinence, without affecting the efficacy of SCCA in alleviating constipation. The rationale for the Jinling procedure includes two main points: firstly, subtotal colectomy eliminates the delayed colonic transit; secondly, the rectal stump the distal mesentery, acting as rectopexy, and therefore the pelvic floor musculature is elevated, which significantly relieves rectocele, intussusception and prolapse.

There are several reasons why we performed another side-to-side cecorectal anastomosis on the basis of SCCA, which successfully relieved the symptoms of ODS. Firstly, the rectal stump 
was fixed together with the distal mesentery after colorectal anastomosis. We assumed that post-operation sterile inflammation developed at the pelvis and formed a pulling force between the rectal stump and the mesentery. The force acted as a rectopexy, which significantly relieved the symptoms of ODS (rectocele, intussusception or prolapse). Secondly, solely end-to-side colorectal anastomosis, although maintaining the ileocecal valve, was often complicated by cecal distention $[10,11]$ and anastomosis stenosis. In the Jinling procedure, however, another side-to-side cecorectal anastomosis was added and the large pouch acted as a reservoir to reduce the occurrence of diarrhea, urgency, and incontinence, which was confirmed by the follow-up results. Twelve patients encountered constipation recurrence after STARR and then received the Jinling procedure in our department. The surgical staples in the lower rectum did not affect the accomplishment of the Jinling procedure because the staple line was re-cut by the linear cutter during the side-to-side anastomosis.

The laparoscopic approach of subtotal colectomy has typical advantages of less invasive surgery: parietal integrity, less postoperative pain and ileus, fewer postoperative adhesions, reduced hospitalization time and, finally, a better cosmesis. Laparoscopic subtotal or total colectomy is criticized as a longer operation compared to the open technique, requiring an ultrasonic or bipolar device (Ligasure) in the surgical armamentarium that facilitates surgical dissection and reducing operative time with safe bleeding control. However, after a learning curve with operations on 40 patients, we finished the laparoscopic section within $95 \mathrm{~min}$ on average (45-135 min).

Different approaches to the colon, numbers of trocars and their position are reported [12-16]. Generally, three trocars are enough to perform a right or left colectomy [12]. The fourth trocar may be necessary in case of difficult dissection of the colonic flexures [15]. Therefore in our mind a subtotal colectomy consists in 2 hemicolectomies plus a segmental resection of the transverse colon, and four trocars are enough. In the surgical treatment of constipation, vascular dissection at the origin is not requested. After adequate identification of the ureters and gonadic vessels, mesenteric dissection may be performed with Ligasure with safe bleeding control, reducing operative time as already reported [17]. The approach to splenic flexure, often high and overlapping, in constipation patients was difficult, especially in obesity [18]. After cutting the gastrocolic ligament, it may be performed from below along the mesentery and afterwards from a lateral approach lifting the colon from the body and tail of the pancreas. In the Jinling procedure, differently from the surgical technique described elsewhere, a $30^{\circ}$ anti-Trendelenburg position was maintained also during the dissection of flexures. In this way the stomach, the greater omentum, the spleen and the liver fall down and the surgeon may pull in front the colon, also performing the mesocolon dissection in a perivisceral plane with clear identification of the duodenum.

An endo-cutter (ECHELON) was used to create a $5-6 \mathrm{~cm}$ side-to-side cecorectal anastomosis in the Jinling procedure. In the beginning, we used the PROXIMATE linear cutter, which had two rows of surgical staples. A high post-surgical anastomosis bleeding rate was observed. Aiming to reduce the bleeding rate, we improved the anastomosis technique and used the ECHELON instead [19], which delivered superior hemostasis through six rows of surgical staples. The anastomosis bleeding rate dropped significantly from about $9 \%$ to $5 \%$ afterwards.

In conclusion, the Jinling procedure is safe for refractory slow-transit constipation associated with outlet obstruction, with minimal major complications and a high satisfaction rate. However, this procedure requires rigorous preoperative examination, exquisite surgical and laparoscopic techniques and excellent perioperative management. The pelvic floor, especially the presacral space, is damaged, and therefore it may be unsalvageable if severe complications, such as anastomosis leakage or ischemia, occur.

\section{Acknowledgments}

Dr. Ding Weiwei and Prof. Jiang Jun contribute equally to this study.

The study is supported by a grant from the Special Project of Clinical High-technology (Grant N.O. 2010gxjs025) and Nanjing Command, PLA (Grant N.O. 12MA081).

\section{References}

1. Talley NJ, Zinsmeister AR, Van Dyke C, Melton LJ 3rd. Epidemiology of colonic symptoms and the irritable bowel syndrome. Gastroenterology 1991; 101: 927-34.

2. Higgins PD, Johanson JF. Epidemiology of constipation in North America: a systematic review. Am J Gastroenterol 2004; 99: 750-9.

3. Schouten WR, de Graaf EJ. Severe, long-standing constipation in adults: indications for surgical treatment. Neth J Surg 1991; 43: 222-9.

4. Sonnenberg A, Koch TR. Epidemiology of constipation in the United States. Dis Colon Rectum 1989; 32: 1-8.

5. Ternent CA, Bastawrous AL, Morin NA, Ellis CN, Hyman NH, Buie WD. Practice parameters for the evaluation and management of constipation. Dis Colon Rectum 2007; 50: 2013-22.

6. Ragg J, McDonald R, Hompes R, Jones O, Cunningham C, Lindsey I. Isolated colonic inertia is not usually the 
cause of chronic constipation. Colorectal Dis 2011; 13 : 1299-302.

7. Wadhawan H, Shorthouse AJ, Brown SR. Surgery for obstructed defaecation: does the use of the Contour device (Trans-STARR) improve results? Colorectal Dis 2010; 12: 885-90.

8. Alame AM, Bahna H. Evaluation of constipation. Clin CoIon Rectal Surg 2012; 25: 5-11.

9. Griffenberg L, Morris M, Atkinson N, Levenback C. The effect of dietary fiber on bowel function following radical hysterectomy: a randomized trial. Gynecol Oncol 1997; 66: 417-24.

10. Todd IP. Constipation: results of surgical treatment. $\mathrm{Br}$ J Surg 1985; 72 Suppl. S12-3.

11. Fasth S, Hedlund H, Svaninger G, Oresland T, Hulten L. Functional results after subtotal colectomy and caecorectal anastomosis. Acta Chir Scand 1983; 149: 623-7.

12. Sample C, Gupta R, Bamehriz F, Anvari M. Laparoscopic subtotal colectomy for colonic inertia. J Gastrointest Surg 2005; 9: 803-8.

13. Kessler H, Hohenberger W. Laparoscopic total colectomy for slow-transit constipation. Dis Colon Rectum 2005; 48: 860-1.

14. Inoue $\mathrm{Y}$, Noro $\mathrm{H}$, Komoda $\mathrm{H}$, et al. Completely laparoscopic total colectomy for chronic constipation: report of a case. Surg Today 2002; 32: 551-4.

15. Bartels SA, Gardenbroek TJ, Ubbink DT, et al. System atic review and meta-analysis of laparoscopic versus open colectomy with end ileostomy for non-toxic colitis. Br J Surg 2013; 100: 726-33.

16. Ehteshami-Afshar S, Nikfar S, Rezaie A, Abdollahi M. A systematic review and meta-analysis of the effects of infliximab on the rate of colectomy and post-operative complications in patients with inflammatory bowel disease. Arch Med Sci 2011; 31: 1000-12.

17. Marcello PW, Roberts PL, Rusin LC, et al. Vascular pedicle ligation techniques during laparoscopic colectomy. A prospective randomized trial. Surg Endosc 2006; 20: 263-9.

18. Marchesi F, Percalli L, Pinna F, et al. Laparoscopic subtotal colectomy with antiperistaltic cecorectal anastomosis: a new step in the treatment of slow-transit constipation. Surg Endosc 2012; 26: 1528-33.

19. Smart CJ, Jones L. Use of Echelon 60 ENDOPATH stapling device in open ileal pouch anal anastomosis (IPAA) surgery. Colorectal Dis 2009; 11: 989. 